\title{
QUANDO A VIDA É FONTE ESSENCIAL DA FORMAÇÃO: Historicidade e Subjetividade
}

\author{
WHEN LIFE IS AN ESSENTIAL SOURCE OF SCIENTIFIC KNOWLEDGE: \\ HISTORICITY AND SUBJECTIVITY
}

Fredy Enrique González*

\begin{abstract}
Resumo
Neste ensaio são apresentadas algumas reflexões preliminares sobre o uso de narrativas na produção do conhecimento científico. Primeiro de tudo, o contexto de emergência deste discurso é levantado. Então, suas características gerais e a necessidade de sua consideração no trabalho científico são brevemente revisadas. Por fim, mostra como o discurso narrativo em matemática poderia ser aplicado, contrastando o hermetismo discursivo das soluções finais, passado para limpo, versus o uso dos Protocolos escritos de resolução de problemas matemáticos.
\end{abstract}

Palavras-chave: Narrativas. Subjetividade. Protocolos Escritos.

\begin{abstract}
In this essay some preliminary reflections are presented regarding the use of narratives in the production of scientific knowledge. First of all, the emergency context of this discourse is raised. Then, its general characteristics and the need for its consideration in scientific work are briefly reviewed. Finally, it shows how the narrative discourse in mathematics could be applied, contrasting the discursive hermeticism of the final solutions, passed to clean, versus the use of the written Protocols of solving mathematical problems.
\end{abstract}

Keywords: Narratives. Subjectivity.Written Protocols.

\section{Resumen}

En este ensayo se presentan algunas reflexiones preliminares relativas al uso de las narrativas en la producción de conocimiento científico. Se plantea, en primer lugar, el contexto de emergencia de este discurso. Luego, se revisan brevemente sus características generales y la necesidad de su consideración en el trabajo científico. Finalmente, se muestra cómo podría ser aplicado el Discurso narrativo en matemática, contrastando el hermetismo discursivo de las soluciones finales, pasadas a limpio, versus el uso de los Protocolos escritos. de resolución de problemas matemáticos.

Palabras Clave: Narrativas. Subjetividad. Protocolos Escritos.

\footnotetext{
Doutor em Educação pela Universidade de Carabobo, Valencia, Venezuela. Membro associado do Comitê Latino-Americano de Matemática Educativa, CLAME. Professor credenciado ao Programa de Pós-Graduação em Educação da Universidade Federal do Rio Grande do Norte. Professor Aposentado na Universidad Pedagógica Experimental Libertado (UPEL, Núcleo Maracay, Aragua, Venezuela). Coordenador-Fundador do Núcleo de Investigación en Educación Matemática "Dr. Emilio Medina" (NIEM; UPEL Maracay) e do Centro de Investigaciones Educacionales Paradigma (CIEP; UPEL Maracay). E-mail: fredygonzalezdem@gmail.com
} 
Quando a vida é fonte essencial da formação: Historicidade e Subjetividade

\section{Introdução}

A narrativa és uma manifestação da insurgência do sujeito na produção de conhecimento cientifico porque esta é "uma construção humana" (ALMEIDA, 2017) $)^{1}$

Assim como "um poeta sem alma equivale a um poema sem palavras" (ALMEIDA, 2017), uma ciência sem sujeitos é como uma cama vazia onde nenhum casal fiz o amor jamais.

$\mathrm{O}$ assunto das narrativas (texto oral, escrito ou vivido) na qual seu autor expõe fatos que ele usa como alicerce de seus argumentos, emergiu no contexto das insatisfações das ciências sociais com os modos hegemônicos, com as heurísticas clássicas e com os instrumentos usados - até agora - na produção de conhecimentos sobre o humano, tanto individual como social.

A defesa do sujeito foi assumida por antropólogos, sociólogos, psicólogos, educadores, epistemólogos, filósofos da ciência, teóricos das organizações... mas também por matemáticos, químicos, físicos, biólogos, y outros representantes das chamadas 'ciências duras'.

Suas ideias, argumentos, arguições, em conjunto, constituem uma chamada para a re-introdução da subjetividade na ciência, a superação do modelo da causalidade determinística dos acontecimentos sociais e individuais, e para o progressivo reconhecimento do papel do sujeito na produção do conhecimento científico.

Por extensão, esse interesse pelo sujeito é ampliado para a reivindicação do local, pessoal, singular, subjetivo, contingente, particular, único, individual e, em geral, para todo aquilo que fica fora da ordem do esperado.

Pode-se dizer, então, que estamos assistindo a um processo de ruptura com as concepções clássicas da ciência, a entrada pela porta grande do sujeito como ator e autor fundamental na produção da ciência, a reivindicação e reconhecimento da subjetividade do sujeito e de

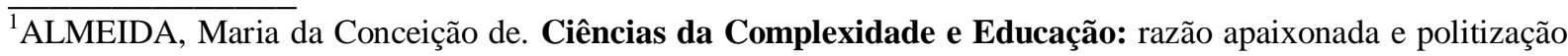
do pensamento. 2.ed. Curitiba: Appris, 2017.
} 
Quando a vida é fonte essencial da formação: Historicidade e Subjetividade

sua integralidade como ser psicossomático que sintetiza nele condições sociais, politicas e culturais.

É Ferrarotti (1988) quem afirma "Toda vida humana se revela até nos seus aspectos menos generalizáveis como uma sintese vertical de uma história social. Todo comportamento ou ato individual nos aprece, até nas formas mais únicas, a sintese horizontal de uma estrutura social." (FERRAROTTI, 1988), ${ }^{2}$

Mas, assim como cada sujeito sintetiza nele a estrutura social, esta é também afetada pelas ações individuais dos sujeitos. É Ferraroti (1988) - de novo - quem acrescenta:

Cada indivíduo não totaliza diretamente uma sociedade global, mas totaliza-a pela mediação de seu contexto social imediato, pelos grupos restritos de que faz parte, pois estes grupos são por sua vez agentes sociais ativos que totalizam o seu contexto, etc. De igual modo, a sociedade totaliza todo indivíduo específico por intermédio de instituições mediadoras que a focalizam cada vez, mas pontualmente para o indivíduo em questão (FERRAROTTI, 1998, apud FREITAS \& GHEDIM, 2015, p. 125). ${ }^{3}$

Por tanto, o sujeito (individual) é construído pela sua sociedade e esta é sintese -pela sua vez- é sintese das ações de aquele assumindoo como produto dela.

Certamente, Almeida (2017) nos diz:

Cientistas e pesquisadores olham (e em geral todos nós olhamos) o mundo a partir do lugar de um observador constituído por sua subjetividade, suas experiências de vida, sua cultura, sua história pessoal. (ALMEIDA, 2017, p. 18) (parênteses e sublinhados adicionados).

\footnotetext{
${ }^{2}$ FERRAROTTI, F. Sobre a autonomia do método biográfico. In: NÓVOA, A; FINGER, M. O método (auto) biográfico e a formação. Lisboa: Ministério da Saúde, 1988. p. 17-34.

${ }^{3}$ FREITAS, Lilliane Miranda \& GHEDIN, Evandro Luiz. Narrativas de formação: origens, significados e usos na pesquisa-formação de professores. Revista Contemporânea de Educacional, v. 10, n. 19, p. 111-131, 2015.
} 
Quando a vida é fonte essencial da formação: Historicidade e Subjetividade

Gostaria de destacar agora as duas expressões que foram sublinhadas na citação anterior: experiência e história pessoal.

Segundo Larrosa (2002)

Tudo aquilo que nos passa, nos acontece, nos toca, que nos afeta como sujeitos, nos deixa marcas (e por tanto) forma e transforma nossa vida dotando-a de singularidade. (LARROSA, 2002, p. 21). ${ }^{4}$

Assim, a experiência é a maneira pela qual os seres humanos se relacionam com a realidade. Por tanto, nós estamos constituídos por nossas experiências e, nesse sentido, somos seres históricos, marcados por nossas circunstancias e a forma como lidamos com elas. Não para aceitá-las como uma fatalidade, senão para desafiá-las mesmo quando nossas possibilidades de superá-las sejam mínimas.

É essa luta contra (e a maioria das vezes dentro) das adversidades que nos rodeiam na que está baseada nossa história pessoal. Por tanto, tudo o que dizemos, escrevemos ou escrevemos é o resultado de nossas experiências.

Mas, nossas experiências pessoais são singulares para cada um de nós. Desse modo, "ninguém pode aprender das experiências de outros, a menos que essas experiências sejam de alguma maneira revivida e tornada como própria" (FREITAS \& GHEDIN, 2015; p. 123). E esse é o papel que as narrativas cumprem: compartilhar, fazer parte dos outros, nossas próprias experiências, lembrando "a in-dissociação existente entre vida e escritura" (ALMEIDA, 2017, p. 256). Isto é, entre historicidade e subjetividade, que são as duas características essenciais das narrativas.

\section{O que são as narrativas?}

Somos um lugar ocupado pelos múltiplos fantasmas que nos habitam. Quase sempre temos a ilusão de nos livrarmos deles e de sermos nós mesmos. Nada é mais ilusório. Nossa voz e nossa

\footnotetext{
${ }^{4}$ LARROSA, Jorge. Notas sobre a experiência e o saber de Experiência. Revista Brasileira de Educação, n. 19, p. 20-28, 2002. Disponível em: http://www.scielo.br/pdf/rbedu/n19/n19a02.pdf. Acesso em: 14 maio 2018.
} 
Quando a vida é fonte essencial da formação: Historicidade e Subjetividade

palavra são apenas um eco distante da marca genética, cognitiva, emocional, histórica e sociocultural com a qual fomos tatuados.

Narrar é uma estratégia sutil e dolorosa com a qual-inutilmente pretendemos nos proteger da hostilidade dos múltiplos mundos que nos habitam.

Uma narrativa é "um complexo conjunto de símbolos que a pessoa usa para conferir significado a seu mundo e a sua vida". Sendo assim, constitui "uma construção discursiva do mundo vivido", uma versão que o sujeito constrói de seu estar no mundo e de si mesmo. Temos, então, que a narrativa vincula "o mundo real" (onde nossa vida acontece) com o "mundo subjetivado". Ou seja, nossas interpretações pessoais do mundo real, a forma como nós o vivemos.

Mas, não se trata só de lembrar coisas, senão de uma organização - a nosso entender - coerente dos fatos que nos aconteceram ao longo da nossa vida: a narrativa dá uma estrutura a nossas experiências. Com ela, nos tentamos reunir e ordenar os diferentes momentos de nossa vida espalhados e dispersos no decurso dos anos. Portanto, uma das dimensões explícitas das narrativas é a temporal, que interage dinamicamente com duas outras: a situacional e a relacional. Em seguida, explicaremos como essa estrutura tridimensional das narrativas opera.

A construção de uma narrativa promove a lembrança do passado, mas com olhos e inquietações do presente e assim e projetar o futuro como possibilidade de transformação e de auto-transformação do sujeito que narra. Assim, o tempo da narrativa se move, embora não linearmente, entre o passado (em um movimento retrospectivo), o presente (em busca da ressignificação dos fatos protagonizados e das experiências vividas) e o futuro (com uma intencionalidade prospectiva).

Por outro lado, a narrativa é um recorte do mundo de vida do narrador. Nesse sentido, ela é construída com base em assim chamadas "recordações-referências" (JOSSO, 2002) $)^{5}$ e que são concebidas

${ }^{5}$ JOSSO, Marie-Christine. Experiências de vida e formação. Lisboa: EDUCA, 2002. 
como aquelas situações que afetam-afetaram ao narrador e que, reflexionadas no momento da escrita narrativa (atual), são carregadas de significados novos pelo narrador-autor-sujeito. Tais situações nos remetem a experiências significativas (acontecimentos marcantes), itinerários, socioculturais, representações ou visões do mundo, crenças, modos de pensar e olhar para o mundo, teorias implícitas, conhecimento tácito, entre outras.

Mas, também, toda narrativa conta uma história que, ao mesmo tempo, é tanto individual quanto social. Esse aspecto da narrativa alude a sua dimensão relacional: toda narrativa é uma história social corporificada.

Temos, então, que a narrativa entrelaça tempo, memória, espaços, conhecimentos e experiências. Entrelaçados, esses elementos dão origem a uma história em que aparecem personagens que são protagonistas de situações sociais (dimensão relacional) que ocorrem em um tempo determinado (dimensão temporal) e em um espaço (dimensão situacional).

A forma como a história é contada constitui um discurso pronunciado em uma linguagem que não é nem técnica, nem neutra, nem objetiva; pelo contrário, está totalmente carregada, cheia da subjetividade do narrador. Então, do ponto de vista discursivo, a narrativa apresenta uma dualidade. Por um lado, há aquilo que é explicitamente declarado (menção de fatos, personagens, situações, etc.). Mas, por outro lado, há o que não é explicitamente declarado e também o que é sentido ou pensado sobre o que foi escrito. Na sua narrativa, o autor fala de si, de seus sonhos, projetos, realizações, fazendo isso ele também pode reconhecer tanto suas fortalezas quanto suas fraquezas e debilidades, embora muitas vezes, estas não sejam reconhecidas

O terceiro componente das narrativas - além do histórico e do discursivo - são as significações atribuídas ao texto. Este componente tem a ver com a interação que é possível estabelecer entre o narrador e seu público. São os membros dessa audiência que dão sentido e significado ao conteúdo do discurso que compõe a história narrada. 
Quando a vida é fonte essencial da formação: Historicidade e Subjetividade

\section{A narrativa cientifica}

A ciência é autobiográfica, pois o objeto, o conhecimento científico dele gerado, é a continuação do sujeito por outros meios, por isso ele é autoconhecimento. (SANTOS, 2002) ${ }^{6}$

Entre a história pessoal do cientista e sua produção de conhecimento, há um elo indissolúvel, indissociável. (ALMEIDA, 2017)

\section{Segundo Almeida (2017)}

A entrada da narrativa nas ciências vai à contramão da objetividade, da neutralidade, da assepsia da ciência pura e dura, própria da racionalidade paradigmática e incontaminada de burocracia do intelecto e da polícia académica ... entre a vida do cientista e a ciência que ele constrói se dá uma simbioses... (ALMEIDA, 2017).

A narrativa científica testemunha a imersão do cientista (sujeito) no universo de pesquisa que acolhe seu assunto de interesse indagatório (investigativo). Assim, a semelhança do escafandrista que olha no fundo do mar, o pesquisador (sujeito-cientista), ao emergir à superfície, conta (narra) sua versão de aquilo que - supostamente - ele conseguiu. Seu desafio não é apenas contar, mas - fundamentalmente encantar, seduzir, cativar.

No discurso científico convencional, até agora hegemônico, o conhecimento produzido exclui seu produtor. Em uma operação meticulosa de alta cirurgia, o cientista deve praticar uma espécie de "eutanásia intelectual" (melhor, um suicídio) que torna viável sua produção pagar as alcavalas alfandegárias impostas pelos carcereiros da academia oficial e, ao fim, poder passar ao Panteão Olímpico da Ciência Oficial.

A primeira coisa a fazer é des-apalavrarse, desaparecer como primeira pessoa e diluir-se numa voz passiva escura, etérea e anônima. Então, ele tem que entrar em um tipo de descanso absoluto que congela seu coração em uma tentativa quimérica de des-emocionarse.

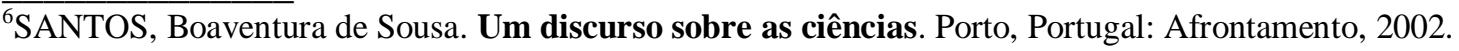


Quando a vida é fonte essencial da formação: Historicidade e Subjetividade

Finalmente, a des-localização total; a anulação do espaço e do tempo; o crime perfeito: não se sabe quem o cometeu, nem como ou quando foi cometido.

No entanto, é inegável que "o conhecimento é uma construção humana" (ALMEIDA, 2017, p.17); portanto, não é possivel dessubjetivá-lo, isto é, excluir o sujeito que o produz.

Assim como todo espetáculo que nos é oferecido à vista, à escuta ou à contemplação, é precedido por inúmeros ensaios de preparação, cheios de emoções, sensações e afetos múltiplas, e também é o caso da produção da ciência. Na geração do conhecimento científico, o cientista coloca em jogo seus afetos, iras, marcas inconscientes, ideologias, valores éticos (ALMEIDA, 2017, p.17) que ele subscreve.

É também inegável que, em nossa relação com o mundo, cada um de nós ocupa uma posição, que se constitui em nosso Lugar Epistemológico (GONZÁLEZ, 2003) ${ }^{7}$ cujas coordenadas de referência são definidas a partir de nossa história pessoal, repleta das experiências que nos permitiram que nos tornamos a pessoa que somos hoje.

Assim, "tudo o que sabemos, sabemos por meio de nossas experiências pessoais" (ALMEIDA, 2017, p. 18), acumuladas a partir do momento em que, com nosso Grito Primário, nós nos abrimos para o mundo do qual fazemos parte inseparável.

Esta inseparabilidade de nós, em relação ao mundo que nos abriga, permeia todas as nossas ações, incluindo, é claro, aquelas que derivam de nossas tarefas profissionais.

Esse vínculo é ativo; somos feitos e, ao mesmo tempo, fazemos nosso mundo, numa relação dialética entre o sócio-estrutural (o que herdamos, nos é dado como língua, cultura, tradições) e o sóciosimbólico (o modo como metabolizamos, cognitivamente e afetivamente, tudo o que nos foi dado e do qual nós idiossincraticamente nos apropriamos). É baseando-nos nessa Matriz Socio-Simbólica como nós nos relacionamos com o mundo. Tal vínculo nos permite ser, não apenas seus espectadores, mas seus (re)criadores.

\footnotetext{
7 GONZÁLEZ, Fredy. Apuntes acerca de algunos conceptos básicos de la investigación cualitativa. Sapiens: Revista Universitaria de Investigación, a. 4, n. 1, p. 107-132, 2003.
} 
Uma das formas de produzir conhecimento sobre o mundo e que se tornou hegemônico é o trabalho científico,

As produções derivadas desta tarefa podem acessar o Panteão Olímpico das Ciências (SNI, PNI, QUALIS, COLCIENCIAS, etc.) se e somente se forem expostas por meio de uma gramática "destituída das marcas de seu autor-sujeito", isto é, "des-subjetivada, fria e supostamente impessoal" (ALMEIDA, 2017, pp. 19-20).

Assim, o discurso da ciência oficial caracteriza-se por privilegiar a exclusão, o desaparecimento, a mudez, o encobrimento, do sujeito que o produz, pretendendo ignorar a indissolúvel "associação entre o sujeito que conhece e as ideias que elabora" (ALMEIDA, 2017, p. 22) e ignorar "a natureza subjetiva da narrativa da ciência" e a "implicação do autor nas palavras, argumentos e textos que materializam a produção do conhecimento científico" (ALMEIDA, 2017, p. 23-25).

Contra essa pretensão, insurgiram, como já foi dito, pensadores proeminentes, como:

Schrödinger (fisico): "se excluímos o sujeito cognoscente da natureza que nos esforçamos para conhecer, retrucamos ao papel de espectador que não pertence ao mundo".

Maturana (biólogo): Tudo o que um observador pode dizer sobre um fenômeno é uma construção que ele elabora a partir de sua relação com o mundo onde tal fenômeno ocorre e de sua visão de mundo e valores.

Bohm (físico): Nossas ideias e conceitos são apoiados com base em uma "infra-estrutura tácita" que é responsável por nossos "desvios de sensibilidade" que às vezes nos dão a alegria da segurança e outros o desconforto da incerteza" (BOHM \& PEAT, 1989, p.282).

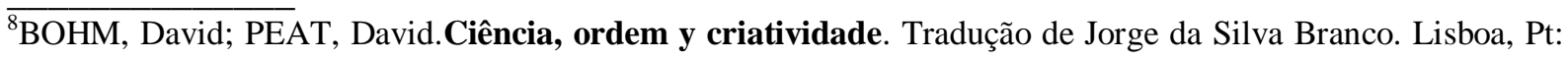
Editorial Gradiva, 1989. 


\section{As narrativas em Matemática}

Em contraste com o hermetismo des-subjetivado do discurso matemático, a narrativa própria dos Protocolos Escritos de Resolução de Problemas Matemáticos é oferecida como uma alternativa. Abaixo está uma amostra de cada um desses discursos (figura 1).

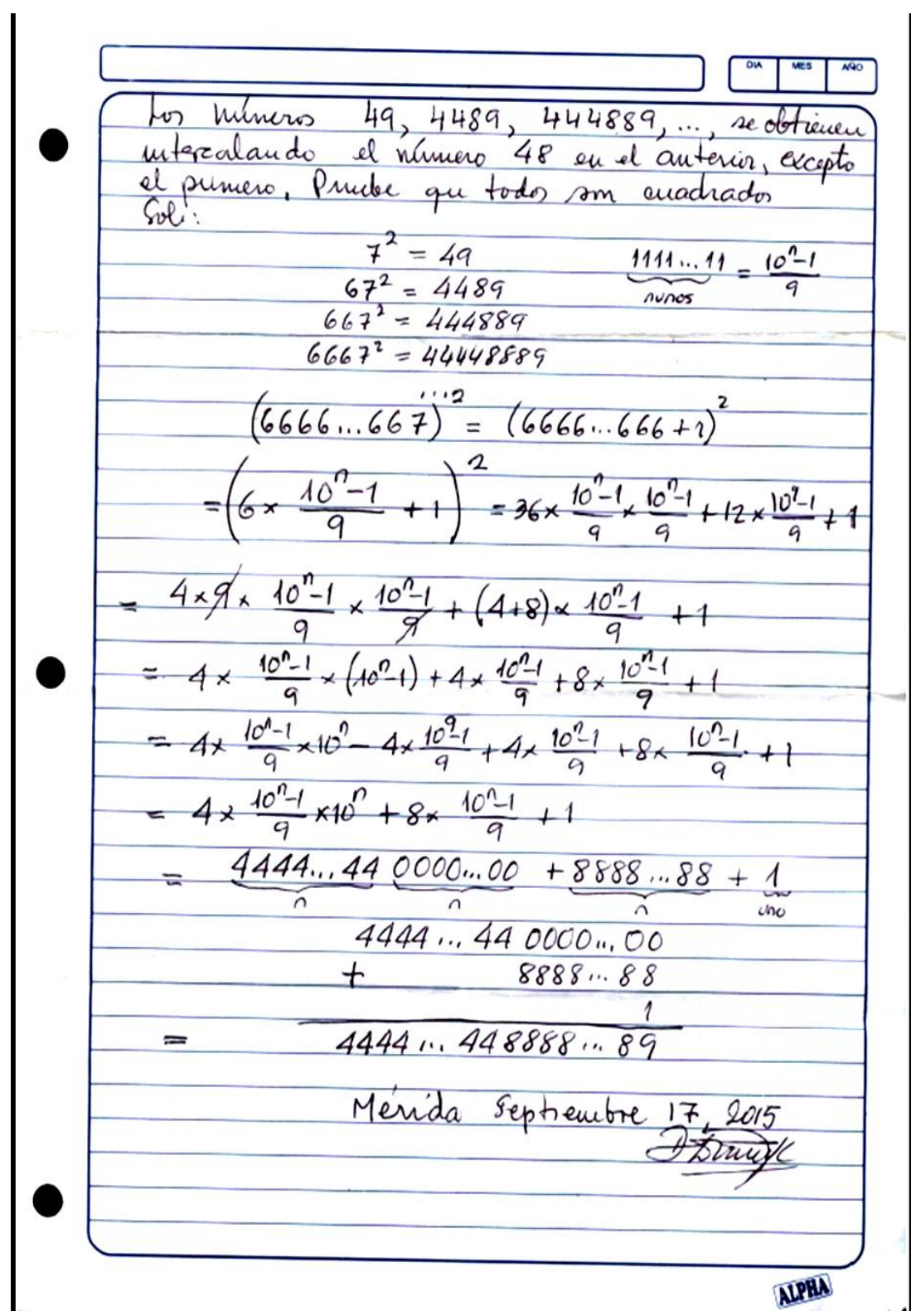

Figura 1- Amostra de discurso.

Fonte: Acervo pessoal 
Quando a vida é fonte essencial da formação: Historicidade e Subjetividade

\section{ANEXO \\ PROTOCOLO \\ TERNAS PITAGÓRICAS}

En un rectángulo, si las longitudes de sus lados y la de su diagonal principal son números enteros, entonces su área es un número múltiplo de doce.

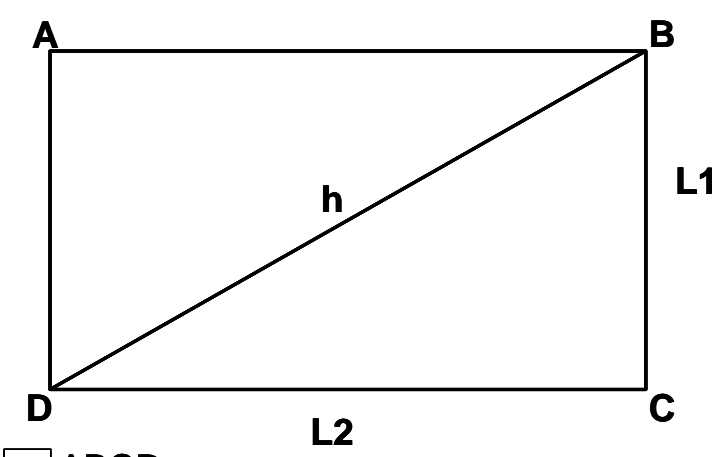

ABCD es un rectángulo cuyas longitudes de sus lados y de su diagonal principal son números enteros

No es la primera vez que me enfrento a este problema; hace algún tiempo, lo discutí con un colega. Sin embargo, en este momento no recuerdo la solución. Por ello, decidí intentar resolverlo apelando a mis propios recursos. De la conversación con el colega (quien sí logró resolverlo) recuerdo que me hizo saber que la solución tenia algo que ver con las "ternas pitagóricas" $x^{2}+y^{2}=z^{2}$,

y que éstas siguen cierta ley de formación; por ello, decidí comenzar tratando de encontrar la ley de formación de las mencionadas ternas. Para hacer esto, partí de la más conocida de ellas $3^{2}+4^{2}=5^{2}$.

Recordé que el colega me había dicho que de los dos sumandos uno tenía base impar y el otro base par; haciendo uso de una calculadora (Casio fx - 3600PA Program 7x), comencé a buscar las ternas tomando el primer sumando con base impar; las que obtuve fueron las siguientes: $3^{2}+4^{2}=5^{2}$; para obtener la segunda procedi por "ensayo y error".

$$
\begin{aligned}
& 5^{2}=25,6^{2}=36 ; 25+36=51 ; 51 \text { no es cuadrado perfecto } \\
& 8^{2}=64 ; 25+64=89 ; 89 \text { no es cuadrado perfecto } \\
& 10^{2}=100 ; 25+100=125 ; 125 \text { no es cuadrado perfecto } \\
& 25+144=169=13^{2} ; 169 \text { sí es cuadrado perfecto }
\end{aligned}
$$


Quando a vida é fonte essencial da formação: Historicidade e Subjetividade

Así que la segunda terna que encontré fue $5^{2}+12^{2}=13^{2}$. El próximo número impar es 7; asociado con éste, comencé con el 8.

$$
\begin{aligned}
& 7^{2}=49,8^{2}=64 ; 49+64=113 ; 113 h \\
& 7^{2}+100^{2}=149 ; 149 \text { no es cuadrado perfecto }
\end{aligned}
$$

Me di cuenta de que se presentan muchos casos; tomando en consideración que el producto de las bases debe ser múltiplo de 12, concluí que había números que, automáticamente, quedaban descartados; por ejemplo, 14 no sirve, porque $7.14=98$ que no es múltiplo de $12 ; 16.7=112$, que tampoco es múltiplo de 12 ; luego, 16 no sirve. $18.7=126$, no es múltiplo de 12; luego, 18 no sirve. Seguí probando. $20.7=140 ; 22.7=154.24 .7=168=$ 12.14 (éste sí es múltiplo de 12), luego, 24 es candidato para formar la terna; veamos: $7^{2}+24^{2}=49+576=625=25^{2}$; luego, la terna es $7^{2}+24^{2}=25^{2}$.

El próximo número impar es 9; los números pares asociados son: 2, 4, $6,8,10,12,14, \ldots$; automáticamente, quedan eliminados $2,6,10,14$, porque $2.9,6.9,10.9,14.9$ no son múltiplos de 12. Candidatos son 4, 8, 12 . Decido ensayar con éstos.

$$
\begin{aligned}
& 9^{2}+4^{2}=81+16=97 ; 97 \text { no es cuadrado perfecto } \\
& 9^{2}+8^{2}=81+64=145 ; 145 \text { no es cuadrado perfecto } \\
& 9^{2}+12^{2}=81+144=225 ; \text { este sí es cuadrado perfecto }
\end{aligned}
$$

Así que otra terna es $9^{2}+12^{2}=225=15^{2}$

El próximo número impar es 11 . Como 11 es un número primo, no divisor de 12; y, además, el producto de las bases debe ser múltiplo de 12, los números pares asociados con 11 que sirven para formar la terna deben ser múltiplos de 12: 12, 24, 36, 48, 60, ..

En este caso, todos los productos posibles son múltiplos de 12; así que debemos ver qué pasa con la suma de los cuadrados:

$11^{2}+12^{2}=265 ;$ no es cuadrado perfecto

$11^{2}+24^{2}=697 ;$ no es cuadrado perfecto

$11^{2}+36^{2}=1417$; no es cuadrado perfecto

$11^{2}+48^{2}=2425$; no es cuadrado perfecto

$11^{2}+60^{2}=3721=61^{2}$; ésta sí es una terna pitagórica: $11^{2}+60^{2}=61^{2}$ 
Quando a vida é fonte essencial da formação: Historicidade e Subjetividade

El próximo número impar es 13; éste es un número primo que tampoco es múltiplo de 12; asíe que de modo análogo a como hice con el 11, los números pares a asociar con 13 son: $12,24,36,48,60,72,84, \ldots$; como en este caso los productos posibles son múltiplos de 12 , debo ver qué pasa con la suma de cuadrados:

$$
\begin{aligned}
& 13^{2}+12^{2}=313 ; \text { no es cuadrado perfecto } \\
& 13^{2}+24^{2}=745 ; \text { no es cuadrado perfecto } \\
& 13^{2}+36^{2}=1465 ; \text { no es cuadrado perfecto } \\
& 13^{2}+48^{2}=2473 ; \text { no es cuadrado perfecto } \\
& 13^{2}+60^{2}=3769 ; \text { no es cuadrado perfecto } \\
& 13^{2}+72^{2}=5353 ; \text { no es cuadrado perfecto } \\
& 13^{2}+84^{2}=7225=85^{2} ; \text { si es cuadrado perfecto }
\end{aligned}
$$

La otra terna es $13^{2}+84^{2}=85^{2}$. Hasta este momento, las ternas que he hallado son:

$$
\begin{aligned}
3^{2}+4^{2} & =5^{2} \\
5^{2}+12^{2} & =13^{2} \\
7^{2}+24^{2} & =25^{2} \\
9^{2}+12^{2} & =15^{2} \\
11^{2}+60^{2} & =61^{2} \\
13^{2}+84^{2} & =85^{2}
\end{aligned}
$$

Observo que cuando el impar es primo, los otros dos números son consecutivos; en cambio, cuando el impar no es primo, los otros números no son consecutivos. Todavia no se me ocurre ninguna relación entre los números; sin embargo, me doy cuenta de que el primer sumando también puede ser par; así que decido buscar algunas ternas cuyo primer sumando tenga base par; de esa manera, al reunir ambos tipos, podría obtener la secuencia ordenada y quizás podría descubrir cierta ley de formación.

Lo que me propongo ahora es buscar ternas de la forma $x^{2}+y^{2}=z^{2}$, donde $x=2 n$, con $n$ entero positivo. Ensayaré con los números 2, 4, 6, 8, 10, 12,14 . Me doy cuenta de que, debido a la propiedad conmutativa de la adición, ya algunas de las ternas están identificadas: $3^{2}+4^{2}=5^{2}, 9^{2}+12^{2}=15^{2}$; como las ternas incluyen un número par y otro impar cuyo producto debe ser múltiplo de 12, hay algunos que queda automáticamente descartados.

Para descartar los impares asociados con el 2, calculo los productos $2.3=6,2.5=10,2.7=14,2.9=18$; tengo la sensación de que son muchos 
Quando a vida é fonte essencial da formação: Historicidade e Subjetividade

los casos que hay que probar y me parece que va a ser muy largo el ensayo con el 2; por eso, intento plantear una ecuación como:

$$
2^{2}+4^{2}=(\mathrm{M} 12) \quad \text { donde M12 significa Múltiplo de } 12
$$

Pero siento que para resolverla precisamente necesito la ley de formación de las ternas que es lo que ando buscando. Así que decido continuar el ensayo en forma sistemática de modo análogo a como lo hice comenzando con un sumando de base impar. El caso de 2, lo dejo para después (subrayo esta frase para que después, al final no se me olvide).

El próximo número par después del 2 es 4 , con éste ya se conoce la terna $\left(3^{2}+4^{2}=5^{2}\right)$. Veré qué pasa con el 6 . Con este se asocian $3,5,7,9$, $11,13,15,17,19,21$. Veamos cuáles se descartan. 3, 5, 7, 9, 11, 13, 15, $17,19,21$ se descartaron todos porque al multiplicar por 6 el resultado no es múltiplo de 12. Decido probar con otros números: 23, 25, 27, 29,31, 33. Llegado a este punto, observo que la única manera de que 6.L sea múltiplo de 12 es que L sea par, como estoy trabajando con L impar, ello es imposible; así que concluyo que en ninguna de las ternas $x^{2}+y^{2}=z^{2}$, donde $x . y=$ M12, puede intervenir el 6, si y es impar.

El próximo número par es 8 . Este es igual a 2.2.2; los números impares con los que ensayaré son $3,5,7,9,11,13,15,17,19,21,23,25,27,29$, 31 y 33.

Como $8=2.4$, para obtener un múltiplo de 12 necesito un número que tenga al 3 como factor; ello permite descartar todos los números impares que no sean múltiplos de 3; "se salvan", entonces, los siguientes: 3, 9, 15, 21, 27 y 33. Decido hacer las verificaciones correspondientes.

$$
\begin{gathered}
8^{2}+3^{2}=64+9=73 \\
8^{2}+9^{2}=145 \\
8^{2}+15^{2}=289 \\
8^{2}+21^{2}=505 \\
8^{2}+7^{2}=793 \\
8^{2}+33^{2}=1153
\end{gathered}
$$

Con ninguno "da", sigo buscando: $35,37,39$; los dos primeros "no sirven" por no ser múltiplos de 3 . Pruebo con $39.8^{2}+39^{2}=1585$, no sirve el 39 . Busco otros múltiplos de 3, mayores que 39, entre ellos están 42, 45, 48, 51.

$$
\begin{aligned}
& 8^{2}+42^{2}=1828 \\
& 8^{2}+45^{2}=2089 \\
& 8^{2}+48^{2}=2368
\end{aligned}
$$


Quando a vida é fonte essencial da formação: Historicidade e Subjetividade

Como "no me dan", comienzo a desesperarme; sin embargo, me calmo y sigo con el ensayo sistemático. $8^{2}+51^{2}=2665$, con 51 tampoco da. Considero otros múltiplos de $3: 3.18=54 ; 3.19=57 ; 3.21=63 ; 3.22=66$.

Cuando iba a poner el siguiente múltiplo de 3 , me doy cuenta de que he estado trabajando con algunos números que automáticamente estaban descartados: los números múltiplos de 3 pero que son, además, números pares (como por ejemplo el 42 y el 48). Tomando en cuente esto, rechazo el 54 y el 66.

$8^{2}+57^{2}=3313 ; 8^{2}+63^{2}=4033$. Pruebo con la calculadora y ninguno me da. Me pregunto: ¿Es que con 8 no da ninguno? ¿Lo mismo que pasa con 6 ? A pesar de esta duda, insisto con 87, 93, 99.

$$
\begin{aligned}
& 8^{2}+87^{2}=7633 \\
& 8^{2}+93^{2}=8713 \\
& 8^{2}+99^{2}=9865
\end{aligned}
$$

Ninguno da.

Sigo buscando. $3.35=105 ; 3.37=111 ; 3.39=117$. Con la calculadora, pruebo con 105 y 111 , no me dan.

Pruebo con $117.8^{2}+117^{2}=64+13689=13753-$ tampoco da.

Cuando estaba en esto y casi me daba por vencido, conversé con una colega y le dije lo de las ternas pitagóricas las cuales, según lo que creía, debían estar formadas por un número impar y uno par; ella me dijo que había una que tenía todos los números pares: 6, 8 y 10. ¡Precisamente la que andaba buscando! $8^{2}+6^{2}=64+36=100=10^{2}$. La misma colega me dijo: "creo que si multiplicamos cualquier terna pitagórica por un número entero, se obtiene otra terna pitagórica. Ensayo a partir de la terna 3, 4, y 5.

\begin{tabular}{c|ccc}
$\mathrm{x}$ & 3 & 4 & 5 \\
\hline $\mathrm{x} 2$ & 6 & 8 & 10 \\
$\mathrm{x} 3$ & 9 & 12 & 15 \\
$\mathrm{x} 4$ & 12 & 16 & 20 \\
$\mathrm{x} 5$ & 15 & 20 & 25
\end{tabular}

En general, esto parece funcionar porque $x^{2}+y^{2}=z^{2} \Rightarrow n x^{2}+n y^{2}=n z^{2}$; siendo $n$ un número entero positivo; así que, si trabajo con números $\mathrm{n}$ que sean cuadrados perfectos obtendré algunas ternas interesantes. Con la primera que pruebo es la que obtengo multiplicando por 5, es decir, 15, 20, 25.

$$
15^{2}+20^{2}=225+400=625=25^{2}
$$


Con la idea que me dio la colega, tengo un criterio sistemático para obtener ternas pitagóricas a partir de la terna inicial $(3,4,5)$. Decido hacer un cuadro para organizar la información.

\begin{tabular}{c|ccc}
$\mathrm{n}$ & $\mathrm{x}$ & $\mathrm{y}$ & $\mathrm{z}$ \\
\hline 1 & 3 & 4 & 5 \\
2 & 6 & 8 & 10 \\
3 & 9 & 12 & 15 \\
4 & 12 & 16 & 20 \\
5 & 15 & 20 & 25 \\
6 & 18 & 24 & 30 \\
7 & 21 & 28 & 35 \\
8 & 24 & 32 & 40 \\
9 & 27 & 36 & 45 \\
10 & 30 & 40 & 50
\end{tabular}

Antes de completar el cuadro, observo que ya había obtenido ternas que no aparecen en el cuadro, por ejemplo la $(5,12,13)$. Decido hacer um cuadro ordenado:

\begin{tabular}{ccc}
$\mathbf{x}$ & $\mathbf{y}$ & $\mathbf{z}$ \\
\hline 3 & 4 & 5 \\
4 & 3 & 5 \\
5 & 12 & 13 \\
6 & 8 & 10 \\
7 & 24 & 25 \\
8 & 6 & 10 \\
9 & 12 & 15 \\
10 & 24 & 26 \\
11 & 60 & 61 \\
12 & 9 & 15 \\
13 & 84 & 85 \\
14 & 48 & 50 \\
15 & 20 & 25 \\
16 & 12 & 20 \\
17 & 144 & 145 \\
18 & 24 & 30 \\
19 & 180 & 181 \\
20 & 15 & 25 \\
21 & 72 & 75 \\
22 & 120 & 122 \\
23 & 264 & 265 \\
24 & 32 & 40 \\
25 & 60 & 65
\end{tabular}


La búsqueda de las ternas en las que intervienen 17, 19 y 23 resultó laboriosa. Por sugerencia de la colega, planteé la siguiente ecuación:

$\mathrm{c}^{2}+17^{2}=(\mathrm{M} 12)^{2}$

Se coloca (M12) porque 17 es un número primo.

$\mathrm{c}^{2}+17^{2}=(\mathrm{M} 12)^{2}=289+144 \mathrm{x}^{2} ;$ así que, $\frac{c^{2}-289}{144}=x^{2}$. Luego de numerosos intentos (ensayo y error) encontré la terna: 17, 144, 145. Por un procedimiento análogo encontré las ternas de $19(19,180,181)$ y $23(23,264,265)$. En el caso de 23, procedí como sigue:

$$
\sqrt{\frac{c^{2}-529}{144}}=x \Rightarrow c^{2}=144 x^{2}+529
$$

Aquí se observa que hay que multiplicar a 144 por un cuadrado perfecto y luego sumarle 529, el resultado debe ser cuadrado perfecto; se le dio valores a $\mathrm{x}$ desde 1 y se fue probando hasta que con $x=22$, se obtuvo:

$$
\begin{aligned}
\mathrm{c}^{2} & =144(22)^{2}+529 \\
& =69696+529 \\
& =70225 \\
& =265^{2}
\end{aligned}
$$

Como $144(22)^{2}=144(22)^{2}=(12.22)^{2}=(264)^{2}$ la terna es 23, 264, 265. Al llegar aquí decido suspender el trabajo para continuarlo después (Hoy es domingo y son las 5:00 PM)

Continuación. Es lunes, reanudo el trabajo, después de haberlo suspendido ayer. Durante este lapso, he estado pensando en el problema pero sin atender a alguna cuestión específica. He pensado en trabajar con el cuadro que contiene las veinticinco primeras ternas pitagóricas con la intención de descubrir alguna relación. Ya, a medida que iba construyendo la tabla, percibí algunas regularidades como, por ejemplo:

a) si uno de los sumandos es un número primo, entonces los otros dos números son consecutivos; 
(b) si el número es compuesto, aparece en tantas ternas como factores primos contenga, con la excepción de que uno de éstos sea 2;

(c) el producto de las bases de los sumandos siempre es múltiplo de 12. Además de esto, no se me ocurre, por ahora, más nada. Voy a trabajar con la suma de los cuadrados para ver qué pasa.

Decido trabajar con las ternas que contienen un número primo como un sumando y formo la tabla siguiente.

\begin{tabular}{ccc}
$\mathbf{x}$ & $\mathbf{y}$ & $\mathbf{z}$ \\
\hline 3 & 4 & 5 \\
5 & 12 & 13 \\
7 & 24 & 25 \\
11 & 60 & 61 \\
13 & 84 & 85 \\
17 & 144 & 145 \\
19 & 180 & 181 \\
23 & 264 & 265
\end{tabular}

Esto es lo que hago.

$\begin{array}{ccc}9+16 & = & 25 \\ 25+144 & = & 169 \\ 36+64 & = & 100 \\ 49+576 & = & 625\end{array}$

No veo nada. Me siento cansado. No se me ocurre nada.

Decido parar (11:02 AM)

\section{CONTINUACIÓN. Martes (5:00 AM)}

Sigo pensando en el problema. Siento que me falta información teórica; por esto, decido buscar un libro que me hable de Teoria de Números. En Hall, M. y Knight, B (1966). Algebra Superior. México: UTEHA, encuentro el siguiente teorema:

"Todo número cuadrado perfecto es de la forma $5 \mathrm{n}$ ó ((5n + 1) ó $(5 n-1) "$ (p. 417); sin embargo, no le veo la relación con mi problema. 
Decido elaborar la siguiente tabla para ver si descubro algo.

\begin{tabular}{cccc}
$\mathbf{x}$ & $\mathbf{y}$ & $\mathbf{z}$ & $x^{2}+y^{2}=z^{2}$ \\
\hline 3 & 4 & 5 & $9+16=25$ \\
4 & 3 & 5 & $16+9=25$ \\
5 & 12 & 13 & $25+144=169$ \\
6 & 8 & 10 & $36+64=100$ \\
7 & 24 & 25 & $49+576=625$ \\
8 & 6 & 10 & $84+36=100$ \\
9 & 12 & 15 & $100+576=676$ \\
10 & 24 & 26 & $121+3600=3721$ \\
11 & 60 & 61 & $144+81=225$ \\
12 & 9 & 15 & $169+7056=7225$ \\
13 & 84 & 85 & $225+400=625$ \\
14 & 48 & 50 & $256+144=400$ \\
15 & 20 & 25 & $289+20736=21025$ \\
16 & 12 & 20 & $324+576=900$ \\
17 & 144 & 145 & $361+32400=32761$ \\
18 & 24 & 30 & $400+2304=2704$ \\
19 & 180 & 181 & $441+5184=5625$ \\
20 & 15 & 25 & $484+14400=14884$ \\
21 & 72 & 75 & $529+69696=70225$ \\
22 & 120 & 122 & $576+1024=1600$ \\
23 & 264 & 265 & $625+3600=4225$ \\
24 & 32 & 40 &
\end{tabular}

No veo nada todavía. Recuerdo que tengo la solución escrita en alguna parte, pero me resisto a buscarla, quiero hallarla por mi mismo

$$
\begin{array}{llllll}
20=2^{2} 5 & 64=2^{3} 8 & 25=5^{2} & 18=2.3^{2} & 16=2^{4} & 8=3^{2} \\
10=2.5 & 12=2^{2} 3 & 14=2.7 & 15=3.5 & 4=2^{2} & 6=2.3 \\
21=7.3 & 22=2.11 & & & &
\end{array}
$$

En Hall y Knight (1966), p. 252, encuentro que un número impar, $(2 n+1)$, puede descomponerse en dos partes enteras, $n$ y $(n+1)$ cuyo producto es máximo. Pienso que me puede ser útil esta información. Decido usarla.

Organizo la información em la siguiente tabla: 


\begin{tabular}{cccccccc}
$\mathbf{x}$ & $\mathbf{2 . n}+\mathbf{1}$ & $\mathbf{n}$ & $\mathbf{n}+\mathbf{1}$ & $\mathbf{y}$ & $\mathbf{z}$ & $x^{2}+y^{2}=z^{2}$ & \\
\hline 3 & $2.1+1$ & 1 & 2 & 4 & 5 & $9+16=25$ & $(2.5-1)+(3.5+1)=5.5$ \\
5 & $2.2+1$ & 2 & 3 & 12 & 13 & $25+44=69$ & $5.5+(9.5-1)=34.5-1$ \\
7 & $2.3+1$ & 3 & 4 & 24 & 25 & $49+576=625$ & $(5.10-1)+(115.5-1)=5.125$ \\
11 & $2.5+1$ & 5 & 6 & 60 & 61 & $121+3600=3721$ & $(5.24+1)+5.720=(744.5+1)$
\end{tabular}

No veo nada.

\section{CONTINUACIÓN (2:00 PM)}

Puedo trabajar sólo con las ternas en las uno de los "catetos" es un número primo, ya que las demás podría obtenerlas a partir de ésta multiplicando por un factor conveniente. Observo que los otros dos son números consecutivos.

Por tanto, planteo la siguiente relación: $(2 n-1)^{2}+(2 m)^{2}=(2 m+1)^{2}$.

Si tengo $x=2 n-1$ puedo obtener $\mathrm{m}$ en función de $\mathrm{n}$.

A partir de la ecuación $(2 n-1)^{2}+(2 m)^{2}=(2 m+1)^{2}$ obtengo que

$$
\begin{gathered}
(2 n-1)^{2}=(2 m)^{2}+2(2 m)+1-(2 m)^{2}=2(2 m)+1=4 m+1 \\
(2 n)^{2}-2(2 n)+1=4 m+1 \\
4 n^{2}-4 n=4 m \\
n^{2}-n=m
\end{gathered}
$$

Ahora voy a verificar si esto funciona. La tabla que antes construí por ensayo y error voy a construirla usando los elementos que acabo de conseguir

\begin{tabular}{cclcc}
$\mathbf{x}$ & $\mathbf{2 . n}-\mathbf{1}$ & $\mathbf{m}=\mathbf{n}^{2}-\mathbf{n}=(\mathbf{n}-\mathbf{1}) \mathbf{n}$ & $\mathbf{y}=\mathbf{2 m}$ & $\mathbf{z}=\mathbf{y}+\mathbf{1}$ \\
\hline 3 & $2.2-1$ & $\mathrm{~m}=4-2=1.2$ & $2.2=4$ & 5 \\
7 & $2.4-1$ & $\mathrm{~m}=16-4=3.4$ & $2.12=24$ & 25 \\
11 & $2.6-1$ & $\mathrm{~m}=5.6=30$ & $2.30=60$ & 61
\end{tabular}

Parece que sí, funciona. Ahora lo que me falta es ver ¿Qué pasa con el producto $\mathrm{x} . \mathrm{y}$ ?, donde $x=(2 n-1), y=2(n-1) n$. Me pregunto si el producto $(2 n-1)[2(n-1) n]$ es múltiplo de doce (M12). Voy a verificar para ciertos casos particulares la siguiente interrogante $\dot{c}(2 n-1)[2(n-1) n]=M 12$ ? 
Quando a vida é fonte essencial da formação: Historicidade e Subjetividade

\begin{tabular}{c|c}
$\mathrm{n}$ & $(2 n-1)[2(n-1) n]$ \\
\hline $\mathrm{n}=2$ & $(2.2-1)[2(2-1) 2]=(4-1) 4=3.4=12$ \\
$\mathrm{n}=3$ & $(2.3-1)[2(3-1) 3]=5.12=\mathrm{M} 12$ \\
$\mathrm{n}=4$ & $(2.4-1)(2.3 .4)=7.24=\mathrm{M} 12$
\end{tabular}

En efecto, para $n=2,3,4$ se cumple. Ahora voy a tratar de probarlo en general, por inducción desde $\mathrm{n}=2$.

Lo que quiero demostrar es que la proposición: $P(n):(2 n-1)[2(n-1) n]=M 12$ es verdadera para $\mathrm{n}=2,3,4, \ldots$

Probaré para $\mathrm{n}=2$

$$
\mathrm{P}(2)=(2.2-1)[2(2-1) 2]=(4-1) 4=3.4=12=\mathrm{M} 12
$$

Por tanto, $\mathrm{P}(2)$ es verdadera. Ahora supongo que $\mathrm{P}(\mathrm{h})$ es verdadera; es decir,

$$
\mathrm{P}(\mathrm{h}) \quad=\quad(2 . \mathrm{h}-1)[2(\mathrm{~h}-1) \mathrm{h}]=\mathrm{M} 12 \text { (Hipótesis de Inducción) }
$$

Quiero demostar que $\mathrm{P}(\mathrm{h}+1)$ es verdadera. en efecto, al sustituir $\mathrm{n}$ por $(\mathrm{h}+$ 1) obtengo

$$
\mathrm{P}(\mathrm{h}+1) \quad=\quad[2 .(\mathrm{h}+1)-1)[2((\mathrm{~h}+1)-1)(\mathrm{h}+1)]
$$

Efectuando las operaciones indicadas obtengo $(2 \mathrm{~h}+1)[2 \mathrm{~h}(\mathrm{~h}+1)]$. Asociando los primeros dos factores, obtengo $(4 h+2) h(h+1)$. Aquí recuerdo que el producto de tres números enteros consecutivos es múltiplo de tres, así que manipulo el primer paréntesis para obtener $[3 \mathrm{~h}+(\mathrm{h}+2)] \mathrm{h}(\mathrm{h}+1)$, y de aquí, por la propiedad distributiva, obtengo $3 h^{2}(h+1)+h(h+1)(h+2)$. Al llegar aquí, "se me tranca el serrucho" porque tengo la suma de dos múltiplos de tres, pero yo ando buscando un múltiplo de 12. No se me ocurre otra cosa que ensayar otra vía a partir de $(2 h+1)[2 h(h+1)]$.

Lo que voy a tratar de hacer es obtener la Hipótesis de Inducción. Veo que, si en el primer paréntesis sumo $0=(1-1)$, obtengo $(2 h+1)=[(2 h-1)+$ 1]; al distribuir me queda lo siguiente:

$$
(2 h-1)[2 h(h+1)]+2 h(h+1)
$$


Voy a introducir otro sumando conveniente

$$
(2 \mathrm{~h}-1)[2 \mathrm{~h}(\mathrm{~h}+1)]+2 \mathrm{~h}(\mathrm{~h}+1)=(2 \mathrm{~h}-1))[2 \underline{(\mathrm{h}+1-1)}(\mathrm{h}+1)]+2 \mathrm{~h}(\mathrm{~h}+1)
$$

El factor subrayado parece que no sirve. Intento otro.

$$
\begin{aligned}
(2 h-1)[2 h(h+1)]+2 h(h+1) & =2 h(h-1)[2 h(h+1-1+1)]+2 h(h+1) \\
& =2 h(h-1)[2 h((h-1)+2)]+2 h(h+1) \\
& =2 h(h-1)[2 h(h-1)]+(2 h-1) 2 h(h-1) 2+2 h(h+1) \\
& =(2 h-1)[2 h(h-1)]+2 h[2(2 h-1)(h-1)+(h+1)]
\end{aligned}
$$

El primer sumando es M12 por ser la Hipótesis de Inducción, así que el problema se reduce a demostrar que el segundo sumando también M12. Eso es lo que voy a tratar de demostrar.

Como

$$
\begin{aligned}
2 h[2(2 h-1)(h-1)+(h+1)] & =2\left(2 h^{2}-2 h-h+2\right)+(h+1) \\
& =4 h^{2}+4 h-2 h+4+h+1 \\
& =4 h^{2}+3 h+5
\end{aligned}
$$

Tengo entonces que

$$
2 \mathrm{~h}[2(2 \mathrm{~h}-1)(\mathrm{h}-1)+(\mathrm{h}+1)]=2 \mathrm{~h}\left(4 \mathrm{~h}^{2}+3 \mathrm{~h}+5\right)
$$

Como este sumando debe ser M12, conjeturo que $\left(4 h^{2}+3 h+5\right)$ debe ser M6. voy a verificar esta conjetura.

$$
\begin{array}{ll}
\mathrm{n}=1 & 4(1)^{2}+3.1+5=12=\mathrm{M} 6 \\
\mathrm{n}=2 & 4(2)^{2}+3.2+5=27 ; \text { no es } \mathrm{M} 6
\end{array}
$$

Este último resultado me obliga a revisar porque tenía casi la certeza de la validez de mi conjetura. Al revisar las operaciones me doy cuenta de un error:

$2(2 \mathrm{~h}-1)(\mathrm{h}+1)$ no es $2\left(2 \mathrm{~h}^{2}-2 \mathrm{~h}-\mathrm{h}+2\right)$ sino que es igual a $2\left(2 h^{2}-2 h-h+1\right)$; así que decido continuar. 


$$
\begin{aligned}
2(2 h-1)(h+1)+(h+1) & =2\left(2 h^{2}-2 h-h+1\right)+(h+1) \\
& =2\left(2 h^{2}-3 h+1\right)+(h+1) \\
& =4 h^{2}-6 h+2+h+1 \\
& ={ }^{4} 4 h^{2}-5 h+3=\text { M6? Conjetura }
\end{aligned}
$$

Al llegar aquí, me doy cuenta de otro error, reviso, $2(2 \mathrm{~h}-1)(\mathrm{h}+1)+(\mathrm{h}+1)=2\left(2 \mathrm{~h}^{2} \ldots\right)$

Decido parar para luego volver sobre el problema (Son las 03:20 PM)

\section{CONTINUACIÓN AL DÍA SIGUIENTE (04:30 PMI)}

Ayer por la tarde, demostré la Tesis de Inducción, ahora me propongo reconstruir esa demostración.

Hipótesis de Inducción

$\mathrm{P}(\mathrm{h})$ es verdadera; es decir,

$\mathrm{P}(\mathrm{h})=(2 . \mathrm{h}-1)[2(\mathrm{~h}-1) \mathrm{h}]=\mathrm{M} 12$ (Hipótesis de Inducción)

Quiero demostrar que $\mathrm{P}(\mathrm{h}+1)$ es verdadera, es decir:

$[2(\mathrm{~h}+1)-1][2((\mathrm{~h}+1)-1)(\mathrm{h}+1)]=\mathrm{M} 12$

En efecto

$$
[2(\mathrm{~h}+1)-1][2((\mathrm{~h}+1)-1)(\mathrm{h}+1)]=[2 \mathrm{~h}+2-1][2 \mathrm{~h}(\mathrm{~h}+1)]
$$

Lo que pretendo es obtener la Hipótesis de Inducción más otro sumando que debe ser múltiplo de 12 .

En el primer corchete está $(2 \mathrm{~h}-1)$ y el $2 \mathrm{~h}$ está en el segundo $[(2 h-1)+2][2 h(h+1)]$ me falta el $(h-1)$; en el $(h+1)$ del segundo corchete puedo agregar $1-1$ y obtener $[(2 \mathrm{~h}-1)+2][2 \mathrm{~h}[(\mathrm{~h}+1)+1-1]]$ Y obtengo que:

(*) $\left.^{*}(2 \mathrm{~h}-1)+2\right][2 \mathrm{~h}[(\mathrm{~h}-1+2]]=[(2 \mathrm{~h}-1)+2][2 \mathrm{~h}(\mathrm{~h}-1)+4 \mathrm{~h}]$

Aplicando la propiedad distributiva obtengo que:

$(* *) P(h+1)=(2 h-1) 2 h(h-1)+2(2 h-1)+4 h(h-1)+4$ 
El primer producto es la Hipótesis de Inducción; por tanto, es múltiplo de 12; sólo falta ver si el resto de la expresión también es múltiplo de 12. Veamos.

$$
2(2 h-1)+4 h(h-1)+4=4 h-2+4 h^{2}-4 h+4=4 h^{2}+2
$$

Aquí hay algo raro. Decido revisar (*).

Creo que en la distributividad me equivoque. En efecto, los errores están en el segundo sumando y en el cuarto sumando del segundo miembro de $(* *) ;$ lo correcto es

$$
(2 h-1) 4 h=8 h^{2}-4 h
$$

así que la expresión a considerar es $(2 h-1) 4 h+4 h(h-1)+8 h$,

la cual, al efectuar las operaciones indicadas, conduce a $8 h^{2}-4 h+4 h^{2}-4 h+8 h$, de la cual resulta $12 \mathrm{~h}^{2}$ que, evidentemente es múltiplo de 12 .

Me queda ahora averiguar cuando uno de los sumandos es par y cuando uno de los sumandos es impar pero no primo. Lo primero que pienso es organizar la información para ver si encuentro algún patrón de comportamiento.

\begin{tabular}{ccccc}
$\mathrm{x}$ & $\mathrm{y}$ & $\mathrm{z}$ & & $2^{3}$ \\
6 & 8 & 10 & 2.3 & 2.5 \\
$2(3$ & 4 & $5)$ & $2\{(2.2-1)+2(2-1) 2=2(2-1) 2+1\}$ \\
\hline & & & \\
10 & 24 & 26 & $2[(2.3-1)+2(3-1) 3=2(3-1) 3+1]$ \\
\hline & & & & \\
20 & 15 & 25 & \\
$2(5$ & 12 & $13)$ & \\
\hline
\end{tabular}

Al llegar aquí se me ocurre plantear lo siguiente

Si $\mathrm{x}=\mathrm{M} p$, donde $\mathrm{M}$ es compuesto $\mathrm{y} p$ es primo $(p=2 n-1$, con $\mathrm{n}$ entero positivo), entonces 


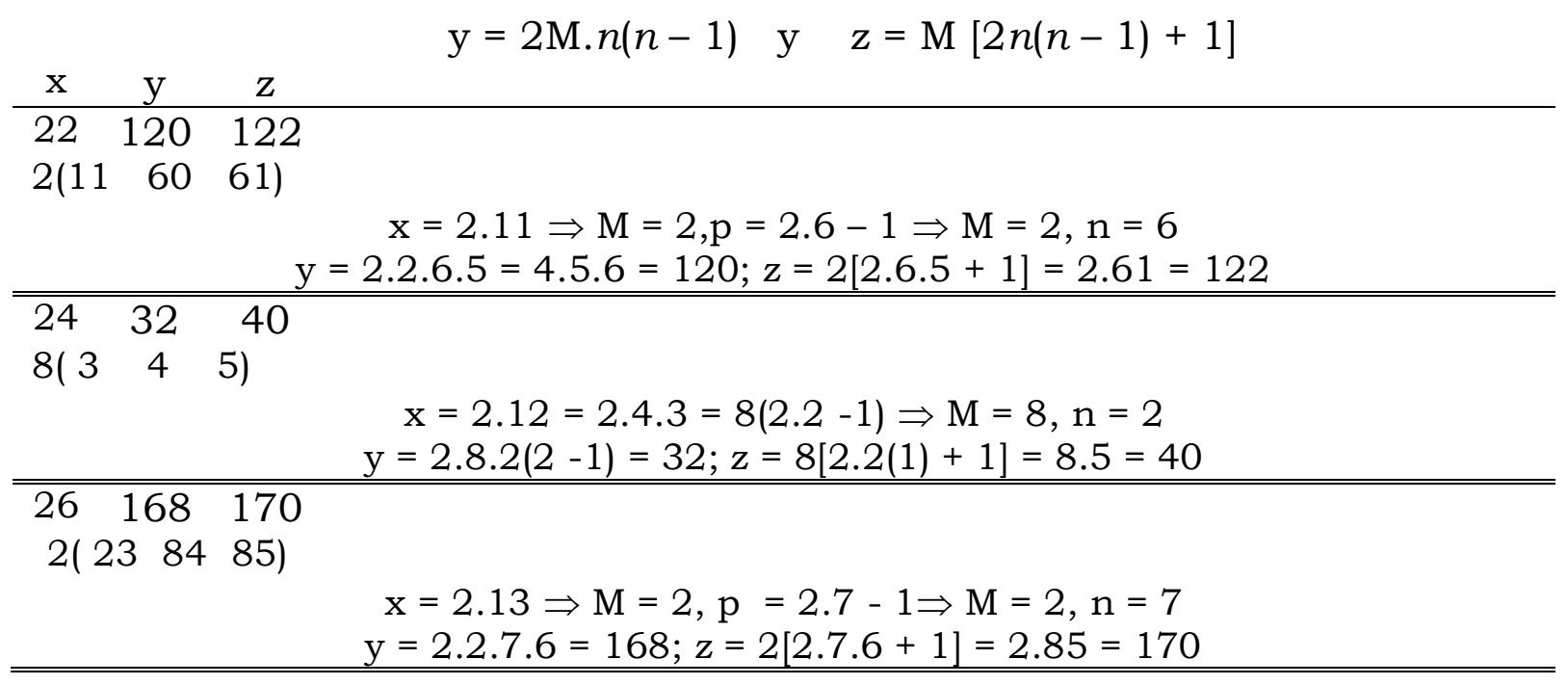

Creo que he encontrado el caso general. Vamos a ver el caso del "impar no primo"; por ejemplo $x=21=7.3$ Aquí observo que se pueden dar dos opciones: $\mathrm{M}=7$ y $\mathrm{p}=3$, ó $\mathrm{M}=3$ y $\mathrm{p}=7$; pienso que deben obtenerse ternas equivalentes.

$$
\begin{aligned}
& M=7, p=3=2 \cdot 2-1 \Rightarrow M=7, n=2 \\
& x^{\prime}=21 \\
& y^{\prime}=2 M n(n-1)=2 \cdot 7 \cdot 2 \cdot 1=28 \\
& z^{\prime}=y^{\prime}+M=35
\end{aligned}
$$

Otro caso: $\mathrm{x}=27=9.3 ; \mathrm{M}=9 ; \mathrm{p}=3=2.2-1 \Rightarrow \mathrm{n}=2$

$$
\begin{aligned}
& y^{\prime}=2 M n(n-1)=2 \cdot 9 \cdot 2(1)=36 \\
& z^{\prime}=y^{\prime}+M=45
\end{aligned}
$$

Pienso que he encontrado el caso general

$[M(2 n-1)]^{2}+[2 M n(n-1)]^{2}=[M[2 n(n-1)+1]]^{2}$; el factor M2 se puede simplificar y queda $[(2 n-1)]^{2}+[2 n(n-1)]^{2}=[[2 n(n-1)+1]]^{2}$ que ya conocemos.

Además $M(2 n-1) \cdot 2 M n(n-1)=M[(2 n-1)(2 n(n-1)))]$ es múltiplo de 12 . Suspendo el trabajo aquí. 
Quando a vida é fonte essencial da formação: Historicidade e Subjetividade

\section{CONTINUACIÓN (TRES DÍAS DESPUÉS)}

Hace varios días que "no trabajo" el problema, he hecho la demostración por inducción de la siguiente proposición $(2 n-1)[2(n-1) n]=\mathrm{M} 12=$ múltiplo de 12. Dicha demostración la he hecho en "papelitos"; mientras cumplía otras obligaciones, pensaba en el problema. Ahora voy a hacerla de nuevo para el caso $\mathrm{n}=\mathrm{h}+1$.

$$
\mathrm{P}(\mathrm{h}+1)=[2(\mathrm{~h}+1)-1][2((\mathrm{~h}+1)-1)(\mathrm{h}+1)]=(2 \mathrm{~h}+1)[2 \mathrm{~h}(\mathrm{~h}+1)]
$$

Aquí estoy pensando en disponer la información de una manera más cómoda que me permita atender a los aspectos estéticos de las demostraciones matemáticas que involucran una secuencia de igualdades. Aquí no recuerdo exactamente cómo lo hice en los "papelitos" (los cuales no tengo a la vista) así que siento que, de nuevo, lo estoy haciendo por primera vez. De nuevo en lo que pienso es en hacer transformaciones para obtener la Hipótesis de inducción, me debe quedar, además, otro sumando que sea múltiplo de 12 . Observando $\mathrm{P}(\mathrm{h}+1)$, me doy cuenta de que $2 \mathrm{~h}$ ya lo tengo pero me falta $(\mathrm{h}-$ 1), pienso obtenerlo sumando y restando 1 , así que me queda:

$$
P(h+1)=[2 h+1][2 h(h+1+1-1)]=(2 h+1)[2 h(h-1)+2]]
$$

Ahora, me falta la expresión $(2 \mathrm{~h}-1)$; trabajaré en el primer factor agregando de nuevo $+1-1$, lo que obtengo es: $P(h+1)=[(2 h-1)+2][2 h(h-1)+2]$; pienso en la distributividad y la aplico

$$
P(h+1)=(2 h-1) 2 h(h-1)+(2 h-1) 2 h 2+2.2 h(h-1)+2.2 h .2
$$

opero

$$
P(h+1)=(2 h-1) 2(h-1) h+8 h^{2}-4 h+4 h^{2}-4 h+8 h
$$

El primer sumando es la Hipótesis de Inducción, en el resto de la expresión algunos de los sumandos se simplifican y queda sólo $12 \mathrm{~h}^{2}$ que es múltiplo de 12; por tanto, $\mathrm{P}(\mathrm{h}+1)=$ Múltiplo de $12+12 \mathrm{~h}^{2}$

Con esto queda resuelto el caso cuando uno de los sumandos es primos, sin embargo, he pensado que falta demostrar analiticamente la relación $(2 n-1)^{2}+[2(n-1) n]^{2}=[2(n-1) n+1]^{2}$; en esto ya he pesado y veo que la demostración es sencilla recurriendo al producto notable "diferencia de cuadrados"; y como dos de los términos son números consecutivos, al hacer transposición de términos las expresiones se simplificarán notablemente.

Voy a proceder. $(2 n-1)^{2}+[2(n-1) n]^{2}=[2(n-1) n+1]^{2}$ es equivalente a:

$$
(2 n-1)^{2}=[2(n-1) n+1]^{2}-[2(n-1) n]^{2}
$$


aquí es donde usaré el producto notable "suma por diferencia igual a diferencia de cuadrados"

$$
\left.(2 n-1)^{2}=[2(n-1) n+1]-[2(n-1) n]\right\}\{[2(n-1) n+1]+[2(n-1) n]\}
$$

El primer factor se reduce a 1 (neutro multiplicativo); por su complejidad pienso en trabajar desde el segundo miembro hacia el primero. Me da la impresión de que será muy fácil.

(A) $\{[2(n-1) n+1]+[2(n-1) n]\}=2 n^{2}-2 n+1+2 n^{2}-2 n$; agrupo términos semejantes y obtengo que:

$(A)=4 n^{2}-4 n+1$; pienso en el producto notable "cuadrado del primero, menos doble producto del primero por el segundo, más cuadrado del segundo", aquí se cumple:

$(A)=4 n^{2}-4 n+1=(2 n)^{2}-2(2 n)+1^{2}=(2 n-1)^{2}$ ilisto! Ahora pienso en hacer un resumen de lo que he encontrado hasta ahora:

\section{Casos}

a. Cuando uno de los sumandos es un número primo

Si $\mathrm{x}=(2 \mathrm{n}-1)$, primo, $\mathrm{n}=$ entero, entonces $\mathrm{y}=2(\mathrm{n}-1) \mathrm{n}, \mathrm{z}=\mathrm{y}+1$

b. Caso general

Si $x=M p$, donde $p=(2 n-1)$ es primo, entonces

$\mathrm{y}=\operatorname{Mn}(\mathrm{n}-1)$,

$\mathrm{z}=\mathrm{M}(\mathrm{y}+1)$

ANTENCIÓN: parece que esto no es cierto.

2. Condiciones a demostrar:

En ambos casos hay que demostrar que $x^{2}+y^{2}=z^{2} \quad$ y $\quad x . y=$ múltiplo de 12

Pienso en variantes del problema pero no se me ocurre ninguna; así que decido parar; quizás después vuelva sobre el problema. 